\title{
Association of Age and Hospitalization Amongst Those with Underlying High-risk Conditions at COVID-19 Diagnosis in a Large, State-wide Health System
}

$\mathrm{J}$ Gen Intern Med 36(9):2906-8

DOI: $10.1007 / \mathrm{s} 11606-021-06942-\mathrm{y}$

(c) The Author(s) 2021

\section{BACKGROUND}

Prior studies have demonstrated increased mortality for older adults and patients with high-risk underlying conditions and COVID-19. ${ }^{1}$ However, the additional likelihood of hospitalization for older adults with these conditions compared to younger patients is unknown.

\section{OBJECTIVE}

The objective of this study was to measure the increased odds of hospitalization for those 65 to 74 years old and $>75$ years old compared to those $<65$ years old with high-risk conditions at COVID-19 diagnosis.

\section{METHODS}

Data for 25,834 patients with SARS-CoV-2 infection, diagnosed by RNA nucleic acid amplification, were analyzed for March 10, 2020, through January 14, 2021, from the University of California COVID Research Data Set (UC CORDS). UC CORDS contains SARS-CoV-2 testing results and COVID-19 treatment information collected from across University of California (UC) Health and is distributed weekly for research purposes. Per UC Health reporting, patients were designated as having a COVID-19-related hospitalization if admitted within 30-days of diagnosis or tested positive during the stay. Presence of high-risk conditions hypertension, hyperlipidemia, heart disease, type 2 diabetes, cancer, asthma/ chronic obstructive pulmonary disorder (COPD), chronic kidney disease (CKD), and end-stage renal disease (ESRD) was determined using the International Classification of Diseases, Tenth Revision, Clinical Modification (ICD-10-CM) codes in the electronic health record (EHR). A combined cardiovascular risk factors category that included patients with hypertension, hyperlipidemia, or heart disease was also assessed. Preexisting conditions included ICD-10-CM codes up to

Received February 2, 2021

Accepted May 20, 2021

Published online June 16, 2021 one-year preceding COVID-19 diagnosis. Multiple logistic regression was used for estimation of odds ratios. Exposure variables were older adult categories defined as 65 to 74 years old and $>75$ years old. Outcome variable was COVID-19related hospitalization. Adjustment variables were gender and race/ethnicity extracted from EHR. All analyses performed in R, version 3.6.3 (R Project for Statistical Computing). Statistical significance set at level of alpha $=0.05$. UC CORDS was operationalized by UC Health as "non-human subjects research" and analyses are considered institutional review board exempt.

\section{FINDINGS}

The mean [SD] age of the study population was 42.4 [20.6] years. The sample was $53 \%$ female, $43 \%$ were White, $22 \%$ non-White Hispanic, 7\% Asian, $6 \%$ Black, and 44\% reported as "Other" (Table 1). For each high-risk condition group, those 65 to 74 years old and $>75$ were at increased odds of hospitalization compared to patients $<65$. All differences between older adult categories and $<65$ reference category were statistically significant except for heart disease and cancer in the 65-

Table 1 Patient Characteristics

\begin{tabular}{ll}
\hline \hline Total number of patients & $25,834(100 \%)$ \\
Hospitalized patients & $4146(16 \%)$ \\
Gender & \\
Female & $13,667(53 \%)$ \\
Race and ethnicity & \\
Non-White Hispanic & $5668(22 \%)$ \\
White & $11,044(43 \%)$ \\
Black & $1482(6 \%)$ \\
Asian & $1859(7 \%)$ \\
Other & $11,449(44 \%)$ \\
Overall mean age & $42.4 \pm 20.5$ \\
Under 65 years old age group & $21,667(84 \%)$ \\
Under 65 years old age group mean & $36.7 \pm 16.2$ \\
Between 65 and 74 years old age group & $2352(9 \%)$ \\
Between 65 and 74 years old age group mean & $69.0 \pm 2.8$ \\
75 years and older age group & $1815(7 \%)$ \\
75 years and older age group mean & $81.8 \pm 4.6$ \\
Conditions & \\
Combined cardiovascular risk factors & $6602(26 \%)$ \\
Hypertension & $4733(18 \%)$ \\
Hyperlipidemia & $4219(16 \%)$ \\
Heart disease & $1667(6 \%)$ \\
Type 2 diabetes & $2972(12 \%)$ \\
Cancer & $1619(6 \%)$ \\
Asthma/COPD & $1973(8 \%)$ \\
Chronic kidney disease & $1504(6 \%)$ \\
ESRD & $562(2 \%)$ \\
\hline
\end{tabular}


$\mathbf{6 5}$ to $\mathbf{7 4}$ years old category compared to younger than 65 years old category
75 years old and above category compared to younger than 65 years old category
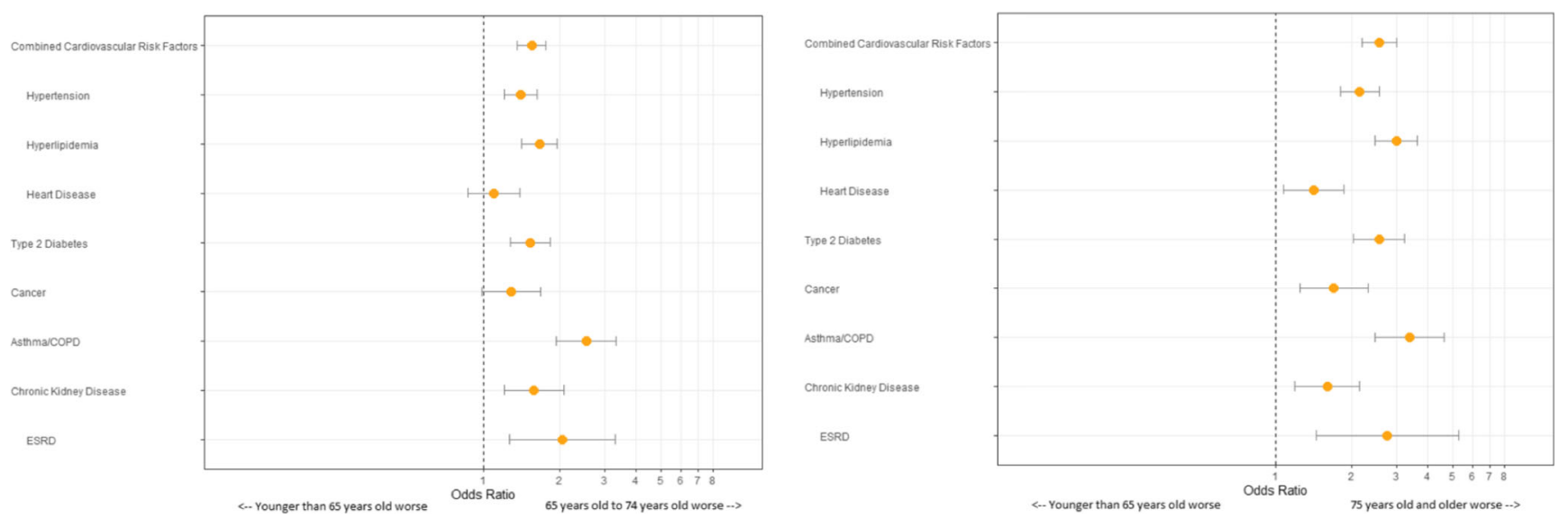

Figure 1 Odds Ratios of Hospitalization Associated with Older Adult Status Category by Comorbidity.

to 74-year-old category. The largest OR was 3.38 [95\% CI, 2.47 to 4.62 ] for patients $>75$ with asthma/COPD. The magnitude in odds of hospitalization for those $>75$ increased to an OR of 2.76 [95\% CI, 1.44 to 5.28] for the ESRD group compared to OR of 1.60 [95\% CI, 1.19 to 2.14] for the CKD group. The OR for patients with either hypertension, hyperlipidemia, or heart disease was 1.55 [95\% CI, 1.36 to 1.77$]$ in 65- to 74-year-olds and 2.56 [95\% CI, 2.19 to 3.01] in those $>75$ (Fig. 1).

\section{DISCUSSION}

In this analysis of COVID-19-positive patients in a large, diverse, state-wide health system, adults $>65$ years with underlying highrisk conditions were much more likely to be hospitalized following SARS-CoV-2 infection than younger patients with high-risk conditions adjusted for gender and race/ethnicity. The observational nature of this study precludes confirmation of a cause and effect relationship between age and hospitalization, but rather demonstrated an association between older age and hospitalization. As of April 15, 2021, COVID-19 vaccination is available to all adults in the USA. ${ }^{2}$ However, a significant portion of the highest risk population (adults $>65$ years old and adults with high-risk conditions) remain unvaccinated accompanied by a troubling slowing rate of vaccination in older adults. ${ }^{3}$ As cases and hospitalizations persist ${ }^{4}$ and highly transmissible SARSCoV-2 variants gain dominance, ${ }^{5}$ outreach efforts to vaccinate these high-risk unvaccinated individuals is critical. We will need to rethink our vaccination strategies to significantly improve convenience and outreach to reach this population. Full vaccination (i.e., 2 doses of the mRNA-based vaccines) of this population is crucial to ensure this most vulnerable population is protected as rapidly as possible. Furthermore, in the high-risk individuals that become infected with SARS-CoV-2, we should prioritize monoclonal antibody therapies, which have shown a benefit in these persons. ${ }^{6}$

Acknowledgements: The authors would like to thank Dr. Kai Zheng and the University of California Irvine support team for the UC COVID19 Research Data Set. Dr. Watanabe is a Member of the National Academies of Sciences, Engineering, and Medicine Forum on Drug Discovery, Development, and Translation and a Scholar in the National Academy of Medicine Emerging Leaders in Health and Medicine Program.

Jonathan H. Watanabe, PharmD, MS, $P h D^{1}$

Jimmy Kwon, $\mathrm{MS}^{2}$

Sanjay R. Mehta, $M D^{3}$

${ }^{1}$ Department of Clinical Pharmacy Practice, School of Pharmacy \& Pharmaceutical Sciences, University of California Irvine,

Irvine, CA, USA

${ }^{2}$ Department of Statistics, Donald Bren School of Information and Computers Sciences, University of California Irvine,

Irvine, CA, USA

${ }^{3}$ Department of Medicine, School of Medicine, University of California San Diego,

La Jolla, CA, USA

Corresponding Author: Jonathan H. Watanabe, PharmD, MS, PhD; Department of Clinical Pharmacy Practice, School of Pharmacy \& Pharmaceutical Sciences, University of California Irvine, Irvine, CA, USA (e-mail: jonathan.watanabe@uci.edu).

Declarations:

Conflict of Interest: Authors have no relevant conflicts of interest to disclose.

Disclaimer: The views expressed in this manuscript do not represent the official views of the National Academies of Sciences, Engineering, and Medicine. 
Open Access This article is licensed under a Creative Commons Attribution 4.0 International License, which permits use, sharing, adaptation, distribution and reproduction in any medium or format, as long as you give appropriate credit to the original author(s) and the source, provide a link to the Creative Commons licence, and indicate if changes were made. The images or other third party material in this article are included in the article's Creative Commons licence, unless indicated otherwise in a credit line to the material. If material is not included in the article's Creative Commons licence and your intended use is not permitted by statutory regulation or exceeds the permitted use, you will need to obtain permission directly from the copyright holder. To view a copy of this licence, visit http://creativecommons. org/licenses/by/4.0/.

\section{REFERENCES}

1. Zhou F, Yu T, Du R, Fan G, Liu Y, Liu Z, et al. Clinical course and risk factors for mortality of adult inpatients with COVID-19 in Wuhan, China: a retrospective cohort study. The Lancet. 2020 Mar 28;395(10229):1054-62.

2. Dooling K. The Advisory Committee on Immunization Practices' Updated Interim Recommendation for Allocation of COVID-19 Vaccine - United
States, December 2020. MMWR Morb Mortal Wkly Rep [Internet]. 2021 [cited 2021 Jan 23];69. Available from: https://www.cdc.gov/mmwr/ volumes/69/wr/mm695152e2.htm

3. CDC. COVID Data Tracker [Internet]. Centers for Disease Control and Prevention. 2020 [cited 2021 Feb 19]. Available from: https://covid.cdc. gov/covid-data-tracker

4. COVID-19 Map [Internet]. Johns Hopkins Coronavirus Resource Center. [cited 2020 Nov 8]. Available from: https://coronavirus.jhu.edu/map.html

5. CDC. New COVID-19 Variants [Internet]. Centers for Disease Control and Prevention. 2020 [cited 2021 Jan 22]. Available from: https://www.cdc. gov/coronavirus/2019-ncov/transmission/variant.html

6. Gottlieb RL, Nirula A, Chen P, Boscia J, Heller B, Morris J, et al. Effect of Bamlanivimab as Monotherapy or in Combination With Etesevimab on Viral Load in Patients With Mild to Moderate COVID-19: A Randomized Clinical Trial. JAMA. 2021 Feb 16;325(7):632.

Publisher's Note: Springer Nature remains neutral with regard to jurisdictional claims in published maps and institutional affiliations. 